\title{
Does Core Stability Training Affect Fundamental Movement Skills in Low Proficiency Children? Evaluation of Performance Process
}

\author{
${ }^{1}$ Abbas Bahram, ${ }^{1}$ Moslem Bahmani", ${ }^{1}$ Farhad Ghadiri \\ ${ }^{1}$ Department of Motor Behavior, Faculty of Sport Sciences, Kharazmi University, Tehran, Iran.
}

\begin{abstract}
The present study investigated the effects of 8-weeks of core stability training on fundamental movement skills in children with low proficiency in both locomotor and object control skills. By using a semi-experimental research design. 30 elementary boy students (means age $=8.89$ years, $\mathrm{SD}=1.06)$ were recruited and organized in training $(\mathrm{n}=$ 15) and control group $(n=15)$. Fundamental movement skills were measured before and after the training period using the test of gross motor development - 2nd (TGMD-2). In comparison with control group, results showed significant improvement in both locomotor $(\mathrm{P}<0.05)$ and object control $(\mathrm{P}<0.05)$ skills in training group. In conclusion, results suggest that core stability training may enhance locomotor and object control performances in children who have low proficiency in fundamental movement skills performance.
\end{abstract}

KEY WORDS: Core Stabilization, Fundamental Movement Skills, Motor Proficiency, TGMD-2, Children.

\section{INTRODUCTION}

Fundamental movement skills (FMS) are elementary forms of movement. FMS are categorized into two major subscales: locomotor skills and object control skills. Locomotor skills are those that involve moving the body from one location to another (e.g. running, jumping, hopping, leaping, galloping, and sliding). Object control skills refers to manipulation and object projection skills (e.g. throwing, kicking, catching, striking, dribbling, and rolling) (1). These skills are considered as the "building blocks" for more advanced (context-specific) skills and form a foundation for various sports and games activities (2). Studies suggest that high proficiency in FMS among school-age children is correlated with many health related benefits such as higher levels of physical activity, cardiovascular fitness, and healthy weight (3-5). In contrast, low proficiency in FMS during childhood is associated with many potentially adverse consequences; a child with poor competency in running, jumping, kicking, catching, throwing, and other basic skills has limited chance at successfully engaging in various sports and activities later in life because he/she will not have "prerequisite skills" to be active (5). Thus, FMS proficiency is an essential factor a to avoid a sedentary lifestyle and prevent weight gain and obesity during childhood and adulthood.

A common misconception is that children "naturally" learn FMS (6); however, research study evidence clearly shows that FMS mastery among children and adolescents is really low (7,

*. Corresponding Author:

Moslem Bahmani

E-mail: bahmani_moslem@yahoo.com 
8) and a considerable number of children do not achieve acceptable levels of these basic skills ( 9 , 10). Therefore, designing appropriate programs and scientific interventions seems necessary to help low FMS-proficient children. Many factors may influence on FMS development; According to dynamic systems approach, motor skill development occurs through the interaction between the task, the organism (learner) and the environment $(11,12)$. Manipulating any of these factors can influence the degree that motor skills develop. Among these factors, within the organism (learner-associated) factors are one of the most important "constraints" that will change outcome of motor performance $(1,13)$. According to Payne and Isaacs (2016) acquiring increased strength, improved balance, and improved motor control causes child patterns in tasks like running, jumping, throwing, etc. to look more adult (13). In the past few years, researchers have paid particular attention to the core training, because it is believed that core region (i.e. lumbo-hip-pelvic complex) has a pivotal role on stabilization and force generation in almost all sports activities (14).

Core stability has been defined as, the ability to control trunk position and motion for the purpose of optimal production, transfer, and control of forces to and from the terminal segments during functional activities (14). Based on Kinetic chain theory, a "break in the chain" should lead to a decrease in optimal force generation or efficiency, and subsequent decrease in performance (15). Biomechanically, all FMS relies on Newton's third law of motion: "for every action, there is an equal and opposite reaction". For example, this law comes into play when a child is trying to perform an overhand throw. The child applies a certain amount of force into the ground, thus the "equal and opposite reaction" occurs, sending the force back through the body, and up through the core (16). Even, if the extremities are strong but the core is weak, then proximal muscles weaknesses will result in an inefficient movement pattern, because the core fails to transfer force to the upper and lower limbs.

No study was found to examine the effect of core stability training on FMS. However, the question of whether core stability exercises have any effect on performance in tasks such as running, jumping, throwing, and kicking have been investigated by several researchers. Saeterbakken, van den Tillaar, and Seiler (2011) examined the effect of core stability training on throwing velocity in female handball players and suggested that core stability training using unstable, closed kinetic chain movements can significantly improve maximum throwing velocity (17). Sharma, Geovinson, and Singh Sandhu (2012) indicated improvement in vertical jump performances of volleyball players after a 9 week core training (18). Stray-Pedersen et al. (2006) reported an improvement in kicking velocity following an 8 -week core stabilization program (19), and Seiler et al. (2006) demonstrated similar effects in maximal clubhead velocity in junior golfers (20). But others like Schilling et al. (2012) (21); Stanton, Reaburn, and Humphries (2004) (22); and Tse, McManus, and Masters (2005) (23) have failed to demonstrate improved performance in tasks like vertical and long jump, agility, 10-yard dash, shuttle run and overhand throwing following core stability training.

With our knowledge, most of previous studies examined the effect of core stability training on athletic performance and little attention been paid to non-athletes. Also, almost all previous studies focused on product of performance (i.e. the outcome of performance has been examined, for example, how long an individual can jump), and no study was found that evaluated the process of performance (i.e. movement pattern or technique, for example, whether arms extended forcefully forward and upward reaching full extension above the head) (6). Therefore, the aim of this study was to investigate the effects of 8-weeks of core stability training on the process of locomotor and object control performance in children with low FMS proficiency.

\section{MATERIALS AND METHODS}

Participants. Initially, 172 elementary school boys ( 7 to 10 years old) were examined under test of gross motor skills development (TGMD2; Ulrich 2000) (6) and then 30 of them who had fewer scores and lower proficiency in FMS performance were selected and randomly divided into two: core stability training $(n=15)$ and the control group $(n=15)$. Research protocol 
and ethics approval was granted by the Faculty of Physical Education and Sport Science of Kharazmi University.

Tools. The test of gross motor development 2nd edition (TGMD-2; Ulrich 2000) was used to assess FMS performance. TGMD-2 is a validated and reliable test (test-retest reliability $=0.88-0.96$ ) that quantitatively assesses the FMS in children between 3 to 10.11 years old. This test was conducted on 3- to 10-year-old Iranian children and internal consistency coefficients for locomotor, object control and gross motor quotient were measured at 0.78 , 0.74 and 0.80 , respectively (24).

This test assesses 12 motor skills in two major subscales: locomotor and object control skills. The six locomotor skills include running, leaping, horizontal jumping, sliding, galloping and hopping. The six object control skills include overhand throwing, kicking, catching, striking, dribbling and underhand ball rolling.

Each motor skill is evaluated according to several performance criteria. If participants demonstrated each criterion the examiner marks a " 1 ", and if they did not demonstrate performance criteria the examiner marks a " 0 ". The item criteria for Horizontal Jump are listed as an example: (1) Preparatory movement includes flexing of both knees with arms extended behind body. (2) Arms extended forcefully forward and upward, reaching full extension above the head. (3) Take off and landing on both feet simultaneously. (4) Arms are thrust downward during landing. Total score for each skill will be computed by adding all scores of the performance criteria (6). Prior to initiation of training period, FMS performance was assessed using TGMD-2. Then, 30 of the participants who had fewer scores in the test were selected and assigned to either core stability training or the control group at random.

Training protocol. Core stability training group received 24 sessions over 8 weeks (3 sessions per week) during the training program. Each session lasted approximately 40-45 minute which included warming up and cooling down phases. Exercises included 3 levels: in Level 1 (weeks 1-2), the purpose of training was mastery of core contraction in which participants practiced static contractions in a stable environment. In Level 2 (weeks 3-5) participants practiced dynamic contraction ina stable environment. Finally, in Level 3 (weeks 5-8) resisted, dynamic movements in unstable environments was applied. To create unstable conditions Swiss balls were used. The core stability training protocol used in this study was based on Jeffrey's suggested protocol (26) (see also Brittenham and Taylor, 2014 for details about core training) (16).

After the end of the training period, all participants were tested with TGMD-2 again. Data was analyzed with $2 \times 2$ ANOVA factorial design (group $\times$ pre-post intervention). Dependent and independent t-test and Bonferroni Post Hoc test were applied within and between group's comparisons. All analyses were conducted using SPSS version 16 and alpha level was set at 0.05 .

\section{RESULTS}

Demographic information of the participant is shown in Table1.

Table1. Demographic information of the participant. Data is shown as $\mathrm{M} \pm \mathrm{SD}$.

\begin{tabular}{ccccc}
\hline Groups & $\begin{array}{c}\text { Age } \\
\text { (years) }\end{array}$ & $\begin{array}{c}\text { Height } \\
(\mathbf{c m})\end{array}$ & $\begin{array}{c}\text { Weight } \\
(\mathbf{k g})\end{array}$ & $\mathbf{N}$ \\
Experimental & $8.83 \pm 1.1$ & $130 \pm 5.5$ & $31 \pm 4.1$ & 15 \\
Control & $8.95 \pm 0.9$ & $132 \pm 5.8$ & $30 \pm 3.7$ & 15 \\
\hline
\end{tabular}

Data analysis for locomotor scores has been shown in Table 2. The results of factorial design indicated that the effect of time (prepost intervention) was significant. This effect suggests that means of locomotor performance has been improved significantly in both groups, from pre-test $($ mean $=13.21)$ to posttest (17.51). The group effect (core stability vs. control) was also significant. The experimental group achieved a higher means score than the control group (20.20 and 14.08 respectively). Interaction (group*time) was also significant, which implies a difference in progress trends from pre-test to post-test in both groups, for this reason, within and between comparisons were used. There was no difference in locomotor performance between experimental and control groups at pre-test $(\mathrm{p}=0.849)$, but after training period, the experimental group showed significant 
improvement in comparison with control group $(\mathrm{p}=0.001)$.

Table 2. Data analysis for locomotor subscale

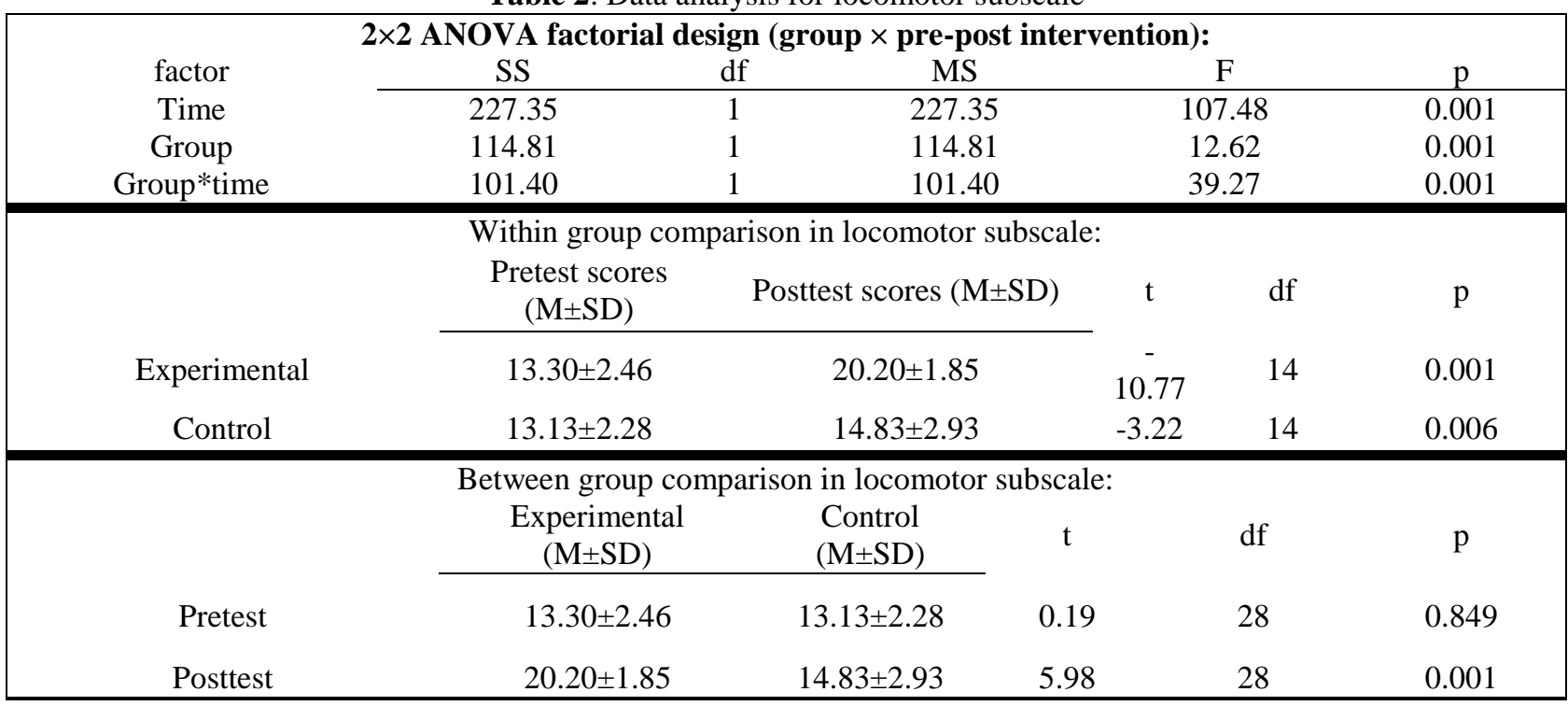

Table 3 shows Data analyses for object control scores. The results demonstrated that time effect was significant. This effect suggests that means of object control performance has been improved significantly in both groups, from pre-test (mean=13.53) to post-test (mean=17.19). Furthermore, the group effect also was significant. This effect indicates that the experimental group achieved a higher means score than the control group (19.93 and 14.40 respectively). Interaction (group $\times$ time) was significant too. This effect shows that from pretest to post-test the progress trends were different in groups, so within and between compactions were used. There was no difference in object control performance between experimental and control groups at pre-test $(\mathrm{p}=0.779)$, but after training the experimental group showed significant improvement in comparison with control group ( $\mathrm{p}=0.001)$.

Table 3. Data analysis for object control subscale

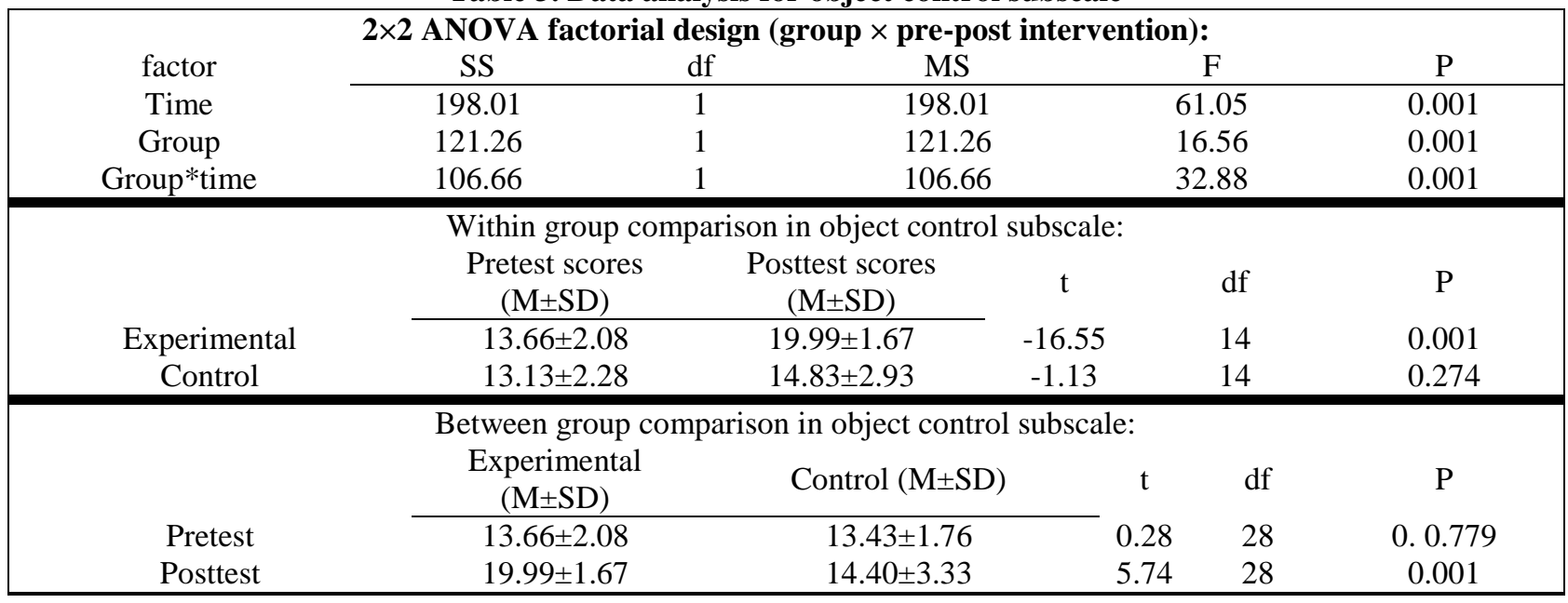




\section{DISCUSSION}

The present study examined the effect of 8weeks of core stability training on locomotor and object control skills in children with low proficiency in FMS. Results indicated that the core stability training group improved significantly in all FMS performance compared with control group.

The finding from this study is in agreement with all other studies that previously indicated core stability training as having a significant effect on physical performance (17-20). For example, a 9-week core training programmed by Prieske et al. (2016) indicated that there were significant improvements in sprint time and kicking performance (25). Our results are also consistent with Stray-Pedersen et al. (2006) which investigated the effect of an 8week core stability training programme on postural balance, kicking velocity and functional strength in elite level soccer players and indicated that these measures increased significantly in the training group compared to the control group (19). However, our findings are inconsistent with Schilling et al. (2013); Stanton, Reaburn, and Humphries (2004); and Tse, McManus, and Masters (2005) (21-23). Tse, McManus, and Masters (2005) examined the effect of 8 -week core endurance training program on college age rowers and found no improvement in the 40-m sprint, overhead medicine-ball throw or2000-m maximum rowing test. The lack of significant differences in some studies may be due to the exercises performed not being functional enough to elicit performance improvement (23). Also, in these studies participants were trained individuals and as such the length or intensity of training may have not been sufficient to elicit a performance enhancement. Core stability training protocol, participant's core endurance and strength in the initiation of the studies, the age of participants, and measuring instruments are additional reasons that may influence I obtained results following core stability training. In the present study we used TGMD-2 which assesses the process of movement, however all previous studies measured the product (outcome) of performance. It is likely that the effects of core stability training on performance process are different from performance product, in other words; improvement in performance process may be easier to attain than performance product. To gain more knowledge in this regard future studies should be conducted.

Based on kinetic chain theory we hypothesized that part of the difficulty in performing some FMS (e.g. running, jumping and hopping) in low proficient children may be due to the core's inability to generate and/or transfer force from lower to upper extremities and vice versa. In addition, powerful rotations are key for those swinging an implement in tasks such as striking, kicking, or overhand throwing. Here, the force from the ground is transferred from a more linear pattern to a strong rotary pattern, resulting in the more powerful and more coordinated movement pattern. From a sport performance perspective, strengthening global muscles (e.g., rectus abdominis, transversis abdominis, internal and external oblique obdominis) provides a foundation for greater torque production and force generation (26) whereas, strengthening the deep muscles provides more stability for the trunk that in turn will create the conditions for better mobility of extremities (14).

It has been suggested that core stability creates several advantages for neural function (26) such as faster nervous system activation, more efficient neural recruitment, improved synchronization of motor units and a lowering of neural inhibitory reflexes. Some authors believe the improvement in performance may be as a result of neural co-ordination and neural recruitment rather than specific improvements in core stability and core strength. It is possible the exercises have influenced the pattern of timing of the muscle activation and force generation $(14,26)$, and therefore significant improvement has occurred in the process of movement performance (i.e., in the movement coordination).

Several researchers have suggested that Swiss ball exercises are most effective for training core stability $(26,27)$; using a Swiss ball provides an unstable surface, which activates the core muscles to a greater extent and improves trunk stability and balance (26). In the present study, exercises progressed from simple static core contraction and slow movements in a stable 
environment to more difficult dynamic movements in an unstable environment (27). With using unstable environments we tried to activate local stabilizer muscles independently and without global muscles co-activation (26). Local muscles which have attachments to spinal vertebrae are associated with the segmental stability and coordination of the core. Therefore, in this study, paying particular attention to local muscles may have been contributed in building the more stable core and thus performance improvements that observed in experimental group.

\section{CONCLUSION}

Although FMS proficiency during childhood is a very important factor to being physically active, maintaining a healthy weight and obesity prevention, many children don't achieve acceptable levels of FMS competency. The results of the present study revealed an 8 -week core stabilization exercises may enhance locomotor and object control performance in children. It seems high strength; endurance and/or coordination in the core region are key factors to improving all FMS in children with low FMS proficiency.

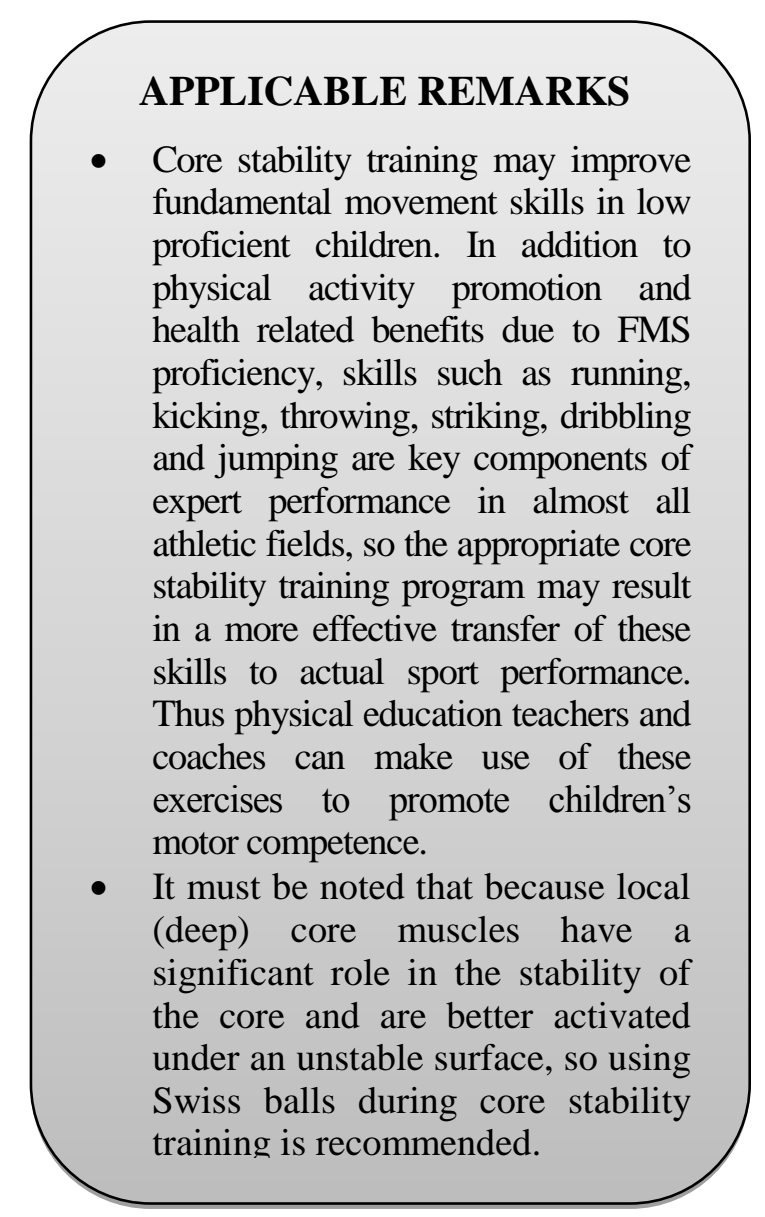

\section{REFERENCES}

1. Haywood K, Getchell N. Development of Human Locomotion. In: Haywood K, Getchell N, editors. Life Span Motor Development. 6th ed: Human Kinetics; 2014.

2. Metcalfe J, Clark JE. The mountain of motor development: A metaphor. In: Clark JE, Humphrey JH, editors. Motor Development: Research and Reviews. Reston, VA: NASPE Pulications; 2002. p. 163-90.

3. Hardy LL, Reinten-Reynolds T, Espinel P, Zask A, Okely AD. Prevalence and correlates of low fundamental movement skill competency in children. Pediatrics. 2012;130(2):e390-8.

4. Lubans DR, Morgan PJ, Cliff DP, Barnett LM, Okely AD. Fundamental movement skills in children and adolescents: review of associated health benefits. Sports medicine (Auckland, NZ). 2010;40(12):1019-35.

5. Stodden DF, Goodway JD, Langendorfer SJ, Roberton MA, Rudisill ME, Garcia C, et al. A Developmental Perspective on the Role of Motor Skill Competence in Physical Activity: An Emergent Relationship. Quest. 2008;60(2):290-306.

6. Ulrich DA. The Test of Gross Motor Development. 2nd ed. TX, USA: Pro-ed, Austin; 2000.

7. Okely $\mathrm{AD}$, Booth ML. Mastery of fundamental movement skills among children in New South Wales: prevalence and sociodemographic distribution. Journal of science and medicine in sport. 2004;7(3):358-72.

8. van Beurden E, Zask A, Barnett LM, Dietrich UC. Fundamental movement skills - How do primary school children perform? The 'Move it Groove it' program in rural Australia. Journal of science and medicine in sport. 2002;5(3):244-52.

9. Goodway JD, Branta CF. Influence of a motor skill intervention on fundamental motor skill development of disadvantaged preschool children. Research quarterly for exercise and sport. 2003;74(1):36-46.

10. Langendorfer SJ, Roberton MA. Individual pathways in the development of forceful throwing. Research quarterly for exercise and sport. 2002;73(3):245-56.

11. Newell KM. Physical constraints to development of motor skills. In: Thomas J, editor. Motor development during preschool and elementary years. Minneapolis: MN: Burgess; 1984. p. 105-20.

Bahram, A., Bahmani, M., Ghadiri, F. (2016). Ann Appl Sport Sci, 4(4): 51-57. 
12. Newell KM. Constraints on the development of coordination. In: Wade MG, Whiting ht, editors. Motor development in children: aspects of coordination and control Springer Netherlands; 1986. p. 341-60.

13. Payne VG, Isaacs LD. Fundamental Locomotion Skills of Childhood. In: Payne VG, Isaacs LD, editors. Human Motor Development: A Lifespan Approach. 9th ed: Routledge; 2016.

14. Kibler WB, Press J, Sciascia A. The role of core stability in athletic function. Sports medicine (Auckland, NZ). 2006;36(3):189-98.

15. Burkhart SS, Morgan CD, Kibler WB. The disabled throwing shoulder: spectrum of pathology Part III: The SICK scapula, scapular dyskinesis, the kinetic chain, and rehabilitation. Arthroscopy : the journal of arthroscopic \& related surgery : official publication of the Arthroscopy Association of North America and the International Arthroscopy Association. 2003;19(6):641-61.

16. Brittenham G, Taylor D. Key Sports Performance Factor. Conditioning to the Core Human Kinetics; 2014.

17. Saeterbakken AH, van den Tillaar R, Seiler S. Effect of core stability training on throwing velocity in female handball players. Journal of strength and conditioning research / National Strength \& Conditioning Association. 2011;25(3):712-8.

18. Sharma A, Geovinson SG, Singh Sandhu J. Effects of a nine-week core strengthening exercise program on vertical jump performances and static balance in volleyball players with trunk instability. The Journal of sports medicine and physical fitness. 2012;52(6):606-15.

19. Stray-Pedersen JI, Magnussen R, Kuffel E, Seiler S, Katch F. Sling exercise training improves balance, kicking velocity and torso stabilization strength in elite soccer players. Medicine and science in sports and exercise. 2006;38(5):S243.

20. Seiler S, Skaanes PT, Kirkesola G, Katch FI. Effects of Sling Exercise Training on Maximal Clubhead Velocity in Junior Golfers: 1781: Board\# 154 2: 00 PM-3: 00 PM. Medicine \& Science in Sports \& Exercise. 2006;38(5):S286.

21. Schilling JF, Murphy JC, Bonney JR, Thich JL. Effect of core strength and endurance training on performance in college students: randomized pilot study. Journal of bodywork and movement therapies. 2013;17(3):278-90.

22. Stanton R, Reaburn PR, Humphries B. The effect of short-term Swiss ball training on core stability and running economy. Journal of strength and conditioning research / National Strength \& Conditioning Association. 2004;18(3):522-8.

23. Tse MA, McManus AM, Masters RS. Development and validation of a core endurance intervention program: implications for performance in college-age rowers. Journal of strength and conditioning research / National Strength \& Conditioning Association. 2005;19(3):547-52.

24.Zarezadeh M, Farokhi A, Kazem Nezhad A. Determining reliability and validity of test of gross motor development (ulrich, 2000) in 3-11 aged children of Tehran city. Olympic. 2011;18(4):85-98 [Article in Farsi].

25. Prieske O, Muehlbauer T, Borde R, Gube M, Bruhn S, Behm DG, et al. Neuromuscular and athletic performance following core strength training in elite youth soccer: Role of instability. Scandinavian journal of medicine \& science in sports. 2016;26(1):48-56.

26. Borghuis J, Hof AL, Lemmink KA. The importance of sensory-motor control in providing core stability: implications for measurement and training. Sports medicine (Auckland, NZ). 2008;38(11):893-916.

27. Santana JC. Hamstrings of Steel: Preventing the Pull, Part II--Training the "Triple Threat". Strength \& Conditioning Journal. 2001;23(1):18. 